
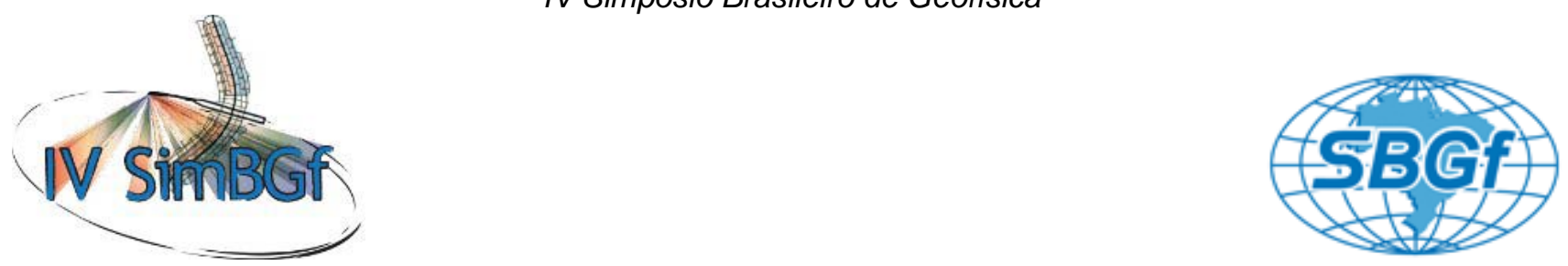

\title{
Realce do gradiente horizontal total de anomalias magnéticas usando a inclinação do sinal analítico. Parte I - Aplicação a dados sintéticos
}

Francisco José Fonseca Ferreira (LPGA/UFPR), Jeferson de Souza (LABAP/UFPR), Alessandra de Barros e Silva Bongiolo (LPGA/UFPR), Luís Gustavo de Castro (LPGA/UFPR), Marco Antonio Thoaldo Romeiro (LABAP/UFPR)

Copyright 2010 SBGf - Sociedade Brasileira de Geofísica

Este texto foi preparado para a apresentação no IV Simpósio Brasileiro de Geofísica Brasília, 14 a 17 de novembro de 2010. Seu conteúdo foi revisado pelo Comitê Técnico do IV SimBGf, mas não necessariamente representa a opinião da SBGf ou de seus associados. É proibida a reprodução total ou parcial deste material para propósitos comerciais sem prévia autorização da SBGf.

\section{Abstract}

We present a method for enhancement of magnetic anomalies which is based on the tilt derivative of the total horizontal gradient. We test its effectiveness by applying it to anomalies generated by rectangular prisms located at different depth and having induced magnetization only and by comparing its results to methods previously reported in literature. The results show that edges enhancement is approximately depth independent. Additionally, our method is less susceptible to noise when compared with methods that also make use of second order derivatives.

\section{Introdução}

Mapas de contorno de anomalias magnéticas (e gravimétricas) são rotineiramente utilizados para delinear falhas e contatos geológicos. Tais mapas encerram sinais com grande variação de amplitude, os quais se superpõem e refletem fontes situadas a várias profundidades, com diferentes geometrias e contrastes de susceptibilidade. Além disso, as anomalias magnéticas observadas dependem da direção da magnetização (induzida e remanente) e da direção de aquisição dos dados. Considerando apenas a magnetização induzida, a técnica de redução ao polo (RTP) permite que as anomalias se posicionem diretamente sobre as fontes, minimizando os efeitos da inclinação magnética. Entretanto, a redução ao polo é reconhecidamente instável em baixas latitudes, uma vez que tanto a parte real quanto a imaginária, ambas no denominador da equação do filtro RTP, se aproximam de zero (e.g. Li, 2008). Por outro lado, em correspondência ao mapeamento magnético, vários métodos foram recentemente divulgados com o objetivo de delinear os limites das fontes das anomalias para facilitar a interpretação. Neste trabalho propomos uma técnica, denominada ISA-GHT, que realça o gradiente horizontal total $(\mathrm{GHT})$ de anomalias magnéticas através da aplicação subsequente da inclinação do sinal analítico (ISA). O método foi testado em dados sintéticos (este trabalho) e reais (Castro et al. 2010, neste simpósio).

Os resultados foram comparados aos obtidos pelos seguintes métodos: amplitude do sinal analítico (ASA Nabighian, 1972,1974; Roest et al., 1992), gradiente horizontal total (GHT - Cordell \& Grauch, 1985), inclinação do sinal analítico (ISA - Miller \& Singh, 1994), gradiente horizontal total da inclinação do sinal analítico (GHT-ISA - Verduzco et al., 2004), Theta map (Wijns et al., 2005) e inclinação do gradiente horizontal total (IGHT - Cooper \& Cowan, 2006).

\section{Métodos}

Os principais métodos empregados na pesquisa estão indicados esquematicamente na Figura 1.

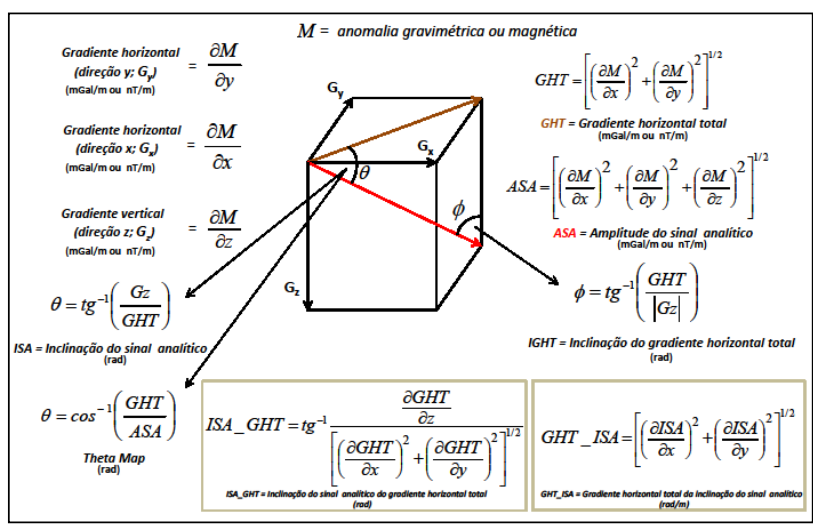

Figura 1 - Principais métodos de realce de anomalias gravimétricas e magnéticas. ASA (ASA - Nabighian, 1972,1974; Roest et al., 1992); GHT (THDR - Cordell \& Grauch, 1985); ISA (TDR, Miller \& Singh, 1994); GHTISA (TDR-THDR - Verduzco et al., 2004); Theta map (Wijns et al., 2005); IGHT (TDX - Cooper \& Cowan, 2006); ISA-GHT (THDR-TDR - Ferreira et al., in prep.).

Anomalias sintéticas oriundas de corpos magnetizados de geometria conhecida são de grande importância na avaliação de métodos de realce. De particular interesse são aquelas decorrentes de modelos prismáticos, em função de suas aproximações às principais estruturas geológicas (Bhattacharyya, 1964; Talwani, 1965; Plouff, 1976; Singh \& Guptasarma, 2001).

Para avaliar o desempenho do método ISA-GHT desenvolvemos o programa PRISMA_GRAV_MAG (Souza et al. em preparação), em linguagem MATLAB ${ }^{\circledR}$, com base na proposta de Bhaskara Rao \& Ramesh Babu (1991), cujos parâmetros de entrada são: inclinação, declinação e intensidade do campo geomagnético (IGRF), coordenadas $x, y$ dos vértices superiores direitos e inferiores esquerdos dos prismas, profundidades ao topo $(z)$ dos modelos, susceptibilidade magnética $(k)$ e inclinação dos corpos em relação ao Norte. 
Os testes foram realizados com base nos modelos 3-D da Figura 2, cujos parâmetros geométricos e magnéticos são indicados nas Tabelas 1 e 2, respectivamente.

De acordo com os dados da Tabela 1, os prismas exibem as mesmas dimensões, porém profundidades ao topo distintas (P1=100m, P2=200m, P3=300m). Conforme a Tabela 2, a magnetização foi considerada induzida $(\mathrm{K}=$ $0.0276 \mathrm{SI}$ ) e os modelos foram inseridos nos ambientes geomagnéticos da cidade de Curitiba-PR e no polo. Para a redução dos dados magnéticos ao polo usamos o algoritmo de Phillips (2007).

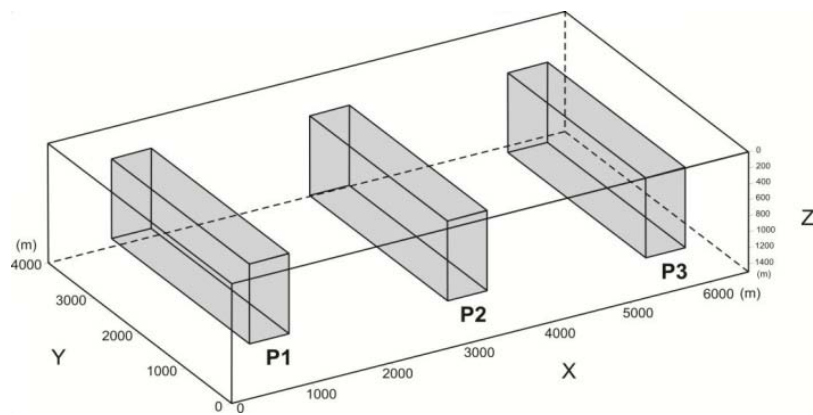

Figura 2 - Representação dos modelos sintéticos 3-D.

Tabela 1 - Parâmetros geométricos dos modelos (Fig. 2).

\begin{tabular}{|c|c|c|c|}
\hline Localização & Prisma P1 & Prisma P2 & Prisma P3 \\
\hline $\mathbf{X}$ & $500-1000$ & $3000-3500$ & $5500-6000$ \\
\hline Y & $500-3500$ & $500-3500$ & $500-3500$ \\
\hline $\mathbf{Z}$ & $100-1100$ & $200-1200$ & $300-1300$ \\
\hline
\end{tabular}

Tabela2 - Parâmetros magnéticos dos modelos (Fig. 2).

\begin{tabular}{|c|c|c|}
\hline Parâmetros & Curitiba & Polo \\
\hline Latitude & $25^{\circ} 25^{\prime} 48^{\prime \prime} \mathrm{S}$ & $85^{\circ} 00^{\prime} 00^{\prime \prime}$ \\
\hline Longitude & $49^{\circ} 16^{\prime} 48^{\prime \prime} \mathrm{W}$ & $132^{\circ} 36^{\prime} 00^{\prime \prime}$ \\
\hline Declinação (Dº) & -18.5 & 0.0 \\
\hline Inclinação $\left(I^{\circ}\right)$ & -35.0 & 90.0 \\
\hline Intensidade (T) & $22789 \times 10^{-9}$ & $56973 \times 10^{-9}$ \\
\hline $\mathrm{J}_{\mathrm{i}}(\mathrm{A} / \mathrm{m})$ & 0.50 & 1.25 \\
\hline
\end{tabular}

\section{Resultados e Discussão}

A Figura 3 mostra as anomalias dos prismas na cidade de Curitiba-PR, na qual se observa a influência da inclinação magnética. A Figura 4 exibe os dados da figura anterior reduzidos ao polo, enquanto a Figura 5 representa as anomalias dos prismas (Fig. 2) situados no polo (Tab. 2). A comparação dos últimos mapas indica que as anomalias, cujas formas são semelhantes, estão posicionadas diretamente sobre os corpos e com máximos em seus centros.

Os resultados discutidos a seguir são decorrentes de transformações dos dados da Figura 4.

A Figura 6, do gradiente horizontal total (GHT, Fig.1), apesar de delinear as bordas dos prismas através de máximos, mostra que as amplitudes de tais sinais são gradativamente atenuadas com a profundidade dos modelos, enquanto seus centros são indicados por mínimos (Cordell \& Grauch, 1985).

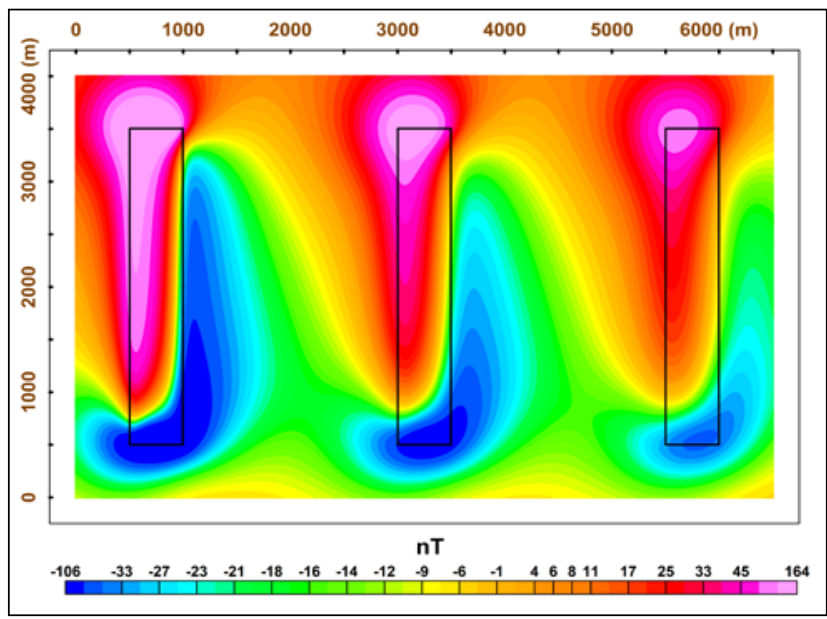

Figura 3 - Mapa de anomalias magnéticas em Curitiba.

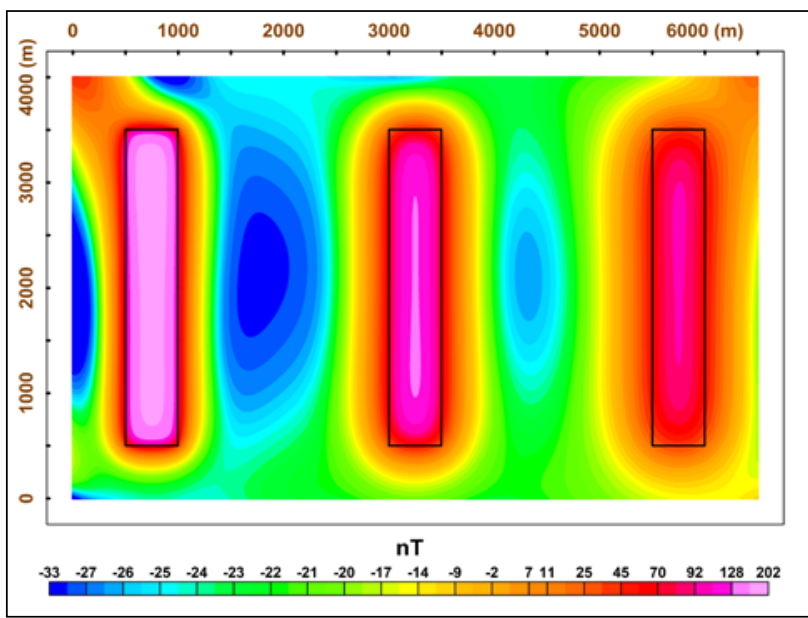

Figura 4 - Mapa de anomalias magnéticas da Figura 3 reduzidas ao polo.

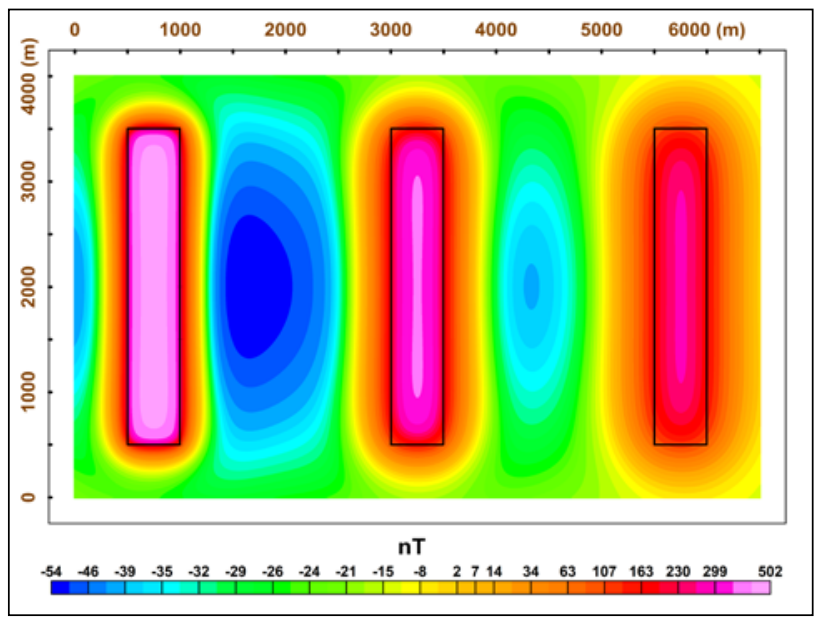

Figura 5 - Mapa de anomalias magnéticas no polo. 


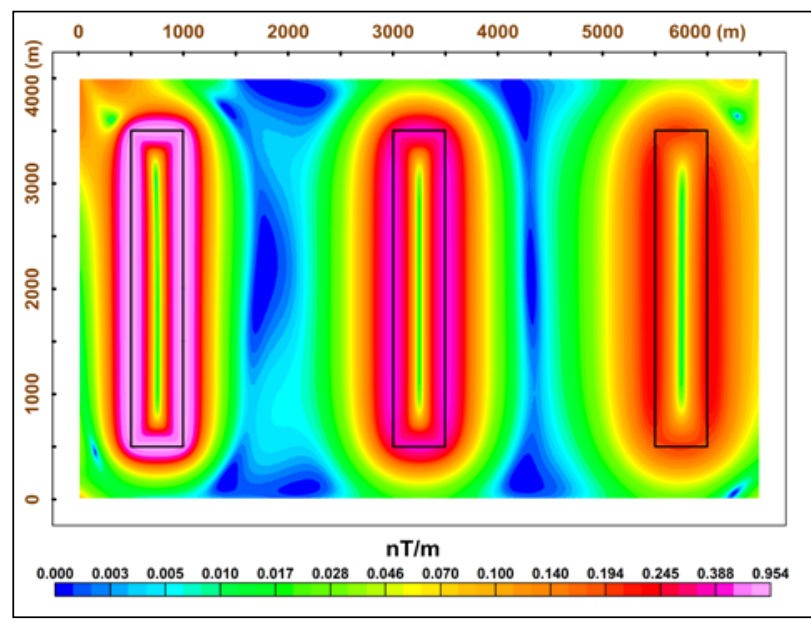

Figura 6 - Mapa do gradiente horizontal total (GHT) calculado com base nos dados da Figura 4.

Fedi \& Florio (2001) apresentaram um método de realce (Enhanced Horizontal Derivative - EHD), cujo cálculo é baseado no GHT de um somatório de derivadas verticais de ordem crescente. Para usar EHD, os dados magnéticos devem ser previamente reduzidos ao polo. Entretanto, do mesmo modo, as amplitudes dos máximos de EHD sobre as bordas das fontes são atenuadas com o incremento de suas profundidades.

Nabighian (1972,1974) aplicou o conceito de sinal analítico a dados de campos potenciais derivados de modelos bidimensionais e sua amplitude foi estendida para corpos 3-D por Roest et al. (1992), com o objetivo de estimar profundidades de fontes magnéticas. Da mesma forma que na Figura 6 , as amplitudes do sinal analítico (ASA, Fig.1) são diminuídas nos prismas mais profundos (Fig. 7). Como se observa na Figura 7, ASA também posiciona as anomalias sobre os corpos e até recentemente foi considerado como não influenciado pela inclinação magnética (Li, 2006a).

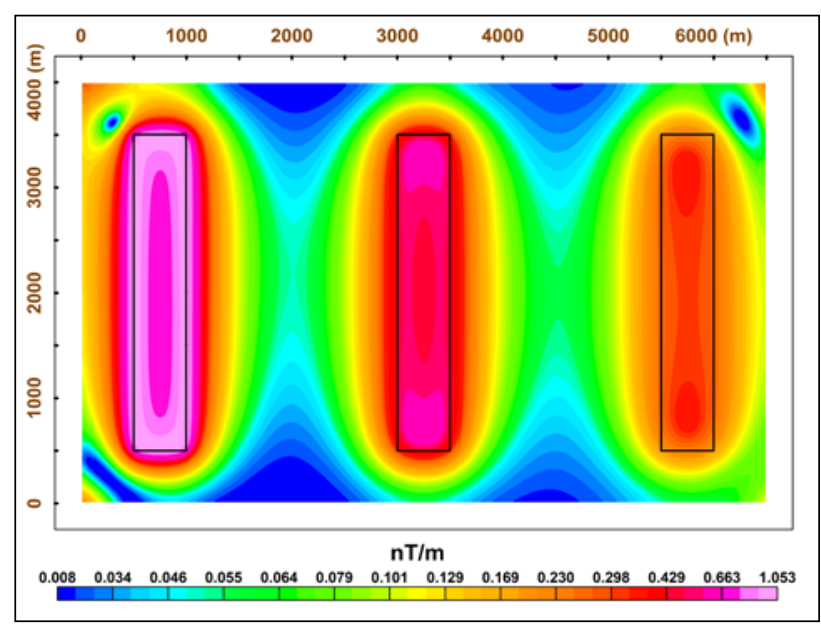

Figura 7 - Mapa da amplitude do sinal analítico (ASA) calculado com base nos dados da Figura 4.

A Figura 8 mostra o mapa da inclinação do sinal analítico (ISA, Fig. 1), introduzido por Miller \& Singh (1994).

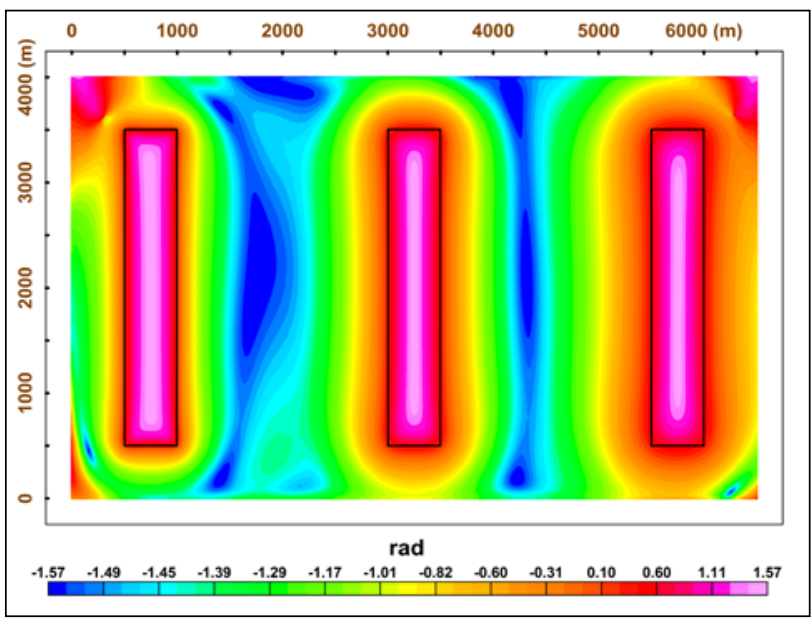

Figura 8 - Mapa da inclinação do sinal analítico (ISA) calculado com base nos dados da Figura 4.

Os principais atributos deste método são equalizar as máximas amplitudes, do que decorre sua independência da profundidade das fontes, e posicioná-las diretamente sobre seus centros (Fig. 8). Este último atributo, entretanto, mostra que ISA não é um método detector de bordas, como assinalaram Cooper \& Cowan (2008). Conforme a Figura 1, ISA normaliza a primeira derivada vertical pelo GHT. Em função das características trigonométricas do arco-tangente, ISA varia de $-\pi / 2$ a $+\pi / 2$ (Fig. 8).

Miller \& Singh (1994), ao avaliarem as respostas de modelos gravimétricos 2-D, notaram que os valores de ISA são positivos (máximos) no centro das fontes, passando à zero nas proximidades de seus limites, em correspondência a valores nulos e máximos respectivamente realçados pelas derivadas vertical e horizontal e vinculados às suas bordas, e negativos (mínimos) fora delas (Fig. 8). Posteriormente, ISA foi revisitado por Verduzco et al. (2004), Salem et al. (2007), Fairhead et al. (2008) e Lahti \& Karinen (2010), dentre outros autores.

O método de Verduzco et al. (2004), amplamente difundido nos últimos anos, consiste, basicamente, da aplicação do gradiente horizontal total (GHT) aos dados da inclinação do sinal analítico (ISA), aqui denominado GHT-ISA. Com base na análise das respostas de perfis S-N perpendiculares ao strike de modelos 2-D (degrau, bloco e dique), inseridos em várias latitudes magnéticas $(0,30,60$ e 90 graus), e em algumas transformações (derivada vertical, GHT e ASA), os autores deduziram que: (i) ASA não varia com a inclinação geomagnética, ao contrário da derivada vertical e GHT. Entretanto, conforme Li (2006a), a primeira constatação só é válida para modelos 2-D de contato vertical e em adição a certos pressupostos (campo potencial medido ao longo do eixo $x$, a partir de uma altura $z$, e gerado por uma fonte disposta paralelamente ao eixo y). Li (2006a) assinalou que em 3-D, mas também de um modo geral em 2-D, ASA depende da direção de indução do campo geomagnético, da direção da magnetização remanente, do mergulho e das profundidades ao topo e a base das 
fontes; (ii) ISA é altamente influenciada pela inclinação e para latitudes magnéticas de $0^{\circ}$ e $90^{\circ}$ seu contorno zero coincide com as bordas dos modelos; (iii) GHT-ISA é independente da inclinação e da intensidade da anomalia magnética, além de realçar e centralizar as máximas amplitudes sobre os limites das fontes. Tais atributos são da maior relevância para a interpretação qualitativa de dados de campos potenciais.

Salem et al. (2007) ratificaram a constatação de Miller \& Singh (1994) e Verduzco et al. (2004), e mostraram que a distância entre os contornos $0^{\circ}$ e $45^{\circ}$ (ou a média da separação do primeiro aos contornos de $\pm 45^{\circ}$ ) é igual a profundidade ao topo de modelos de contato vertical. Esta técnica, denominada Tilt-depth por Salem et al. (2007), só é válida para dados reduzidos ao polo, uma vez que ISA é dependente da inclinação magnética (Verduzco et al., 2004). Portanto, considerando tais pressupostos, profundidades estatísticas de fontes podem ser derivadas diretamente de ISA, uma vez que é possível medir, automaticamente, as distâncias do contorno zero a qualquer outro.

Fairhead et al. (2008) citaram algumas limitações do método de Salem et al. (2007), resultantes da influência de outros corpos 2-D e 3-D e de seus mergulhos, de variações laterais de susceptibilidade magnética (K), da presença de magnetização remanente e de anomalias compostas oriundas da proximidade de fontes e propuseram um outro (Tilt-Depth-Dip- $\Delta K$ ) que contempla o mergulho, o contraste de $\mathrm{k}$ e as estimativas de erro das profundidades dos modelos.

Lahti \& Karinen (2010) fizeram uso de ISA em correspondência a vários níveis de continuação para cima do campo magnético previamente reduzido ao polo, de modelos prismáticos verticais e com mergulho, para delinear os limites de corpos reais em subsuperfície, com base nos seus respectivos contornos zero.

Comparamos a seguir os métodos GHT-ISA (Verduzco et al., 2004) e ISA-GHT. O mapa da Figura 9 exibe os resultados da aplicação da técnica GHT-ISA (Fig. 1), onde se observa que os limites dos corpos são bem marcados por acentuadas amplitudes, embora, de forma semelhante a alguns dos métodos antes discutidos (e.g. GHT, Fig. 6), elas se atenuam em função das profundidades dos corpos. Pelo contrário, os resultados do método ISA-GHT (Fig. 1), indicados na Figura 10, mostram que as bordas dos prismas são igualmente realçadas por máximas amplitudes do sinal, como decorrência da equalização pelo ISA, portanto sem guardar relação com a profundidade dos modelos.

Considerando que os dois últimos métodos utilizam derivadas de segunda ordem, os dados da Figura 4 foram contaminados por um ruído Gaussiano com um desvio padrão de $0.1 \mathrm{nT}$, conforme a Figura 11, a partir dos quais foram empregadas as técnicas GHT-ISA (Fig. 12) e ISA-GHT (Fig. 13). A comparação de tais figuras mostra que o método de Verduzco et al. (2004) é mais sensível a ruídos do que ISA-GHT.

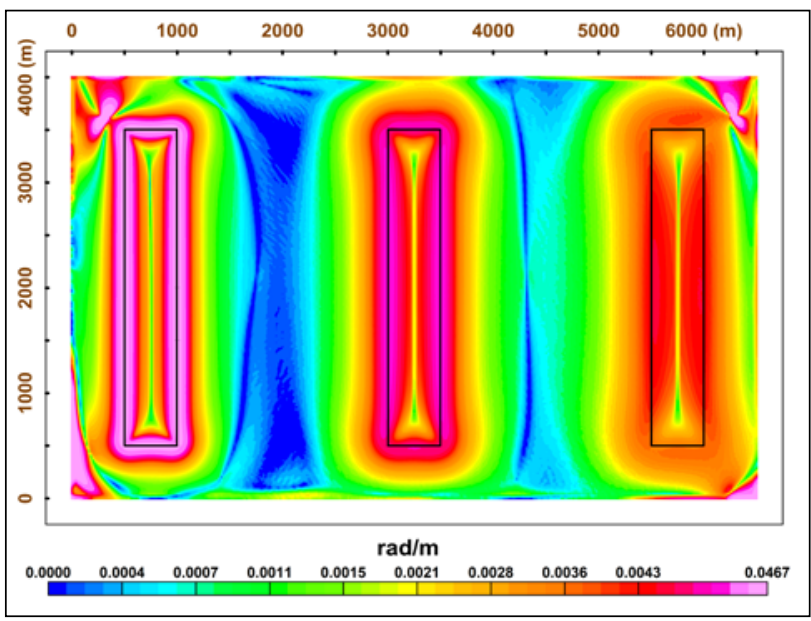

Figura 9 - Mapa do gradiente horizontal total da inclinação do sinal analítico (GHT-ISA) calculado com base nos dados da Figura 4.

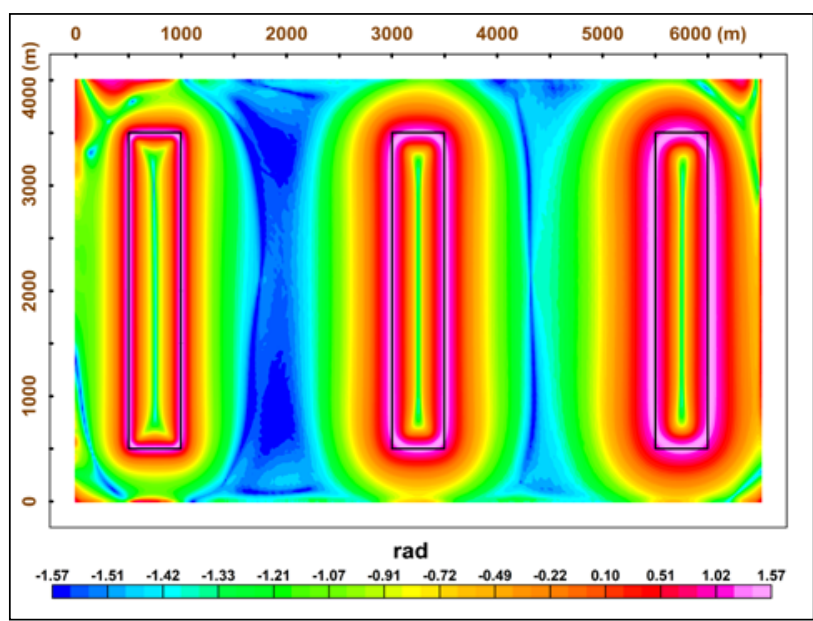

Figura 10 - Mapa da inclinação do sinal analítico do gradiente horizontal total (ISA-GHT) calculado com base nos dados da Figura 4.

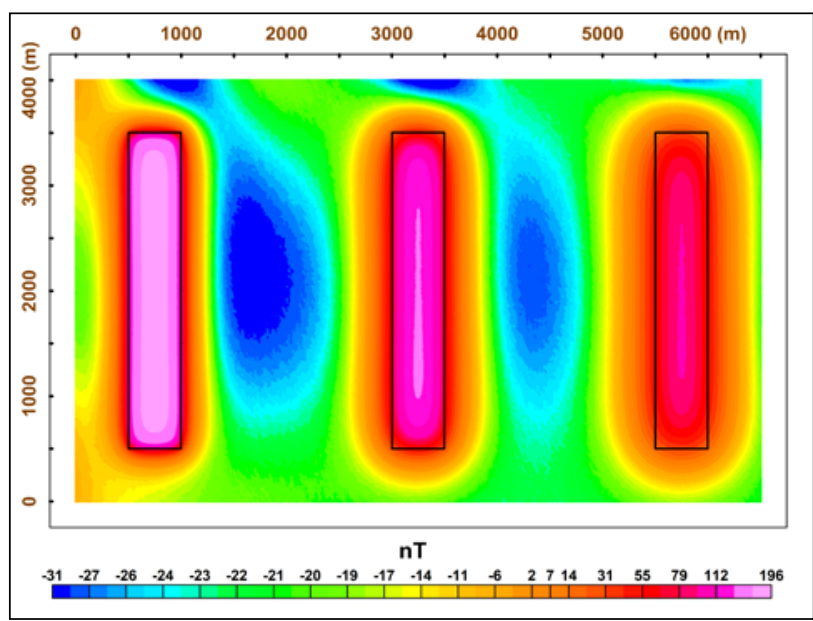

Figura 11 - Mapa de anomalias magnéticas da Figura 4 (Curitiba-RTP) contaminado por um ruído Gaussiano com um desvio padrão de $0.1 \mathrm{nT}$. 


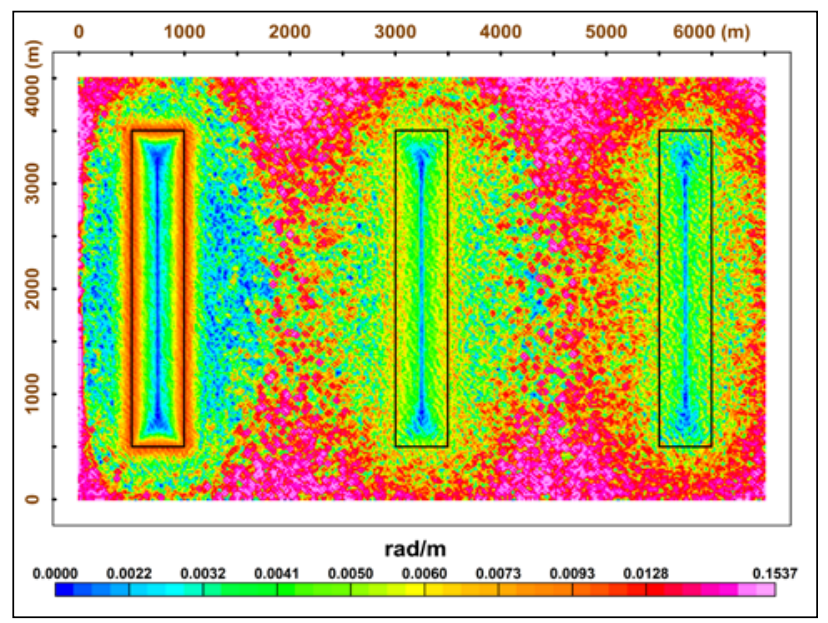

Figura 12 - Mapa do gradiente horizontal total da inclinação do sinal analítico (GHT-ISA) calculado com base nos dados da Figura 11.

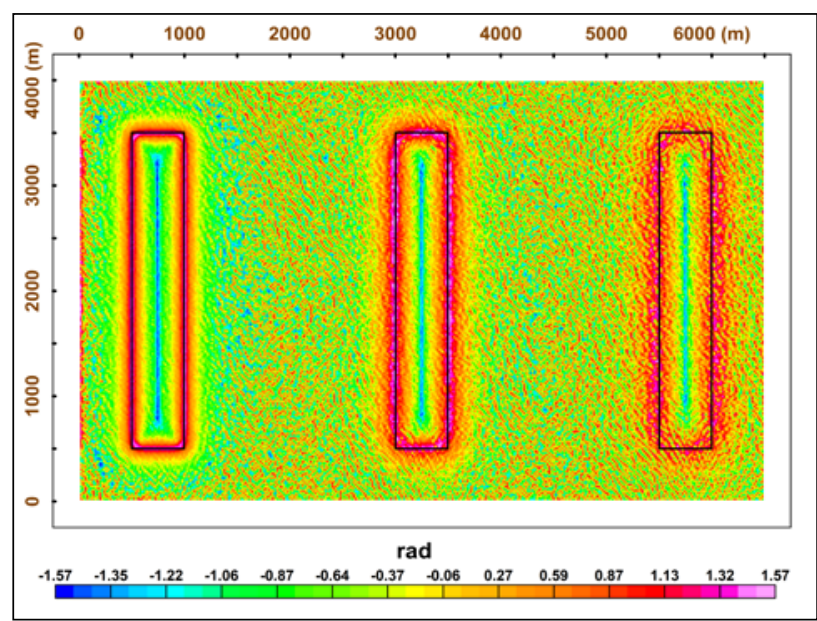

Figura 13 - Mapa da inclinação do sinal analítico do gradiente horizontal total (ISA-GHT) calculado com base nos dados da Figura 11.

As relações de ISA, Theta map e IGHT são igualmente importantes. As Figuras 14 e 15 ilustram os resultados de Theta map (Wijns et al., 2005) e IGHT (Cooper \& Cowan, 2006), respectivamente. Ambos, assim como ISA, são métodos de normalização de derivadas. Enquanto o primeiro utiliza ASA para normalizar GHT, o segundo recorre à derivada vertical (valores absolutos) para normalizar GHT (Fig. 1).

Theta map e IGHT são equivalentes e os sinais contrários decorrem da complementaridade de $\theta$ e $\varnothing$ (Fig. 1), ou seja, enquanto o primeiro delineia as bordas e os eixos dos prismas por mínimos e máximos, respectivamente, o inverso se verifica no segundo (Figs. 14 e 15). As duas últimas figuras revelam também que as assinaturas vinculadas às bordas dos prismas, apesar de próximas, não se posicionam diretamente sobre seus limites, por denotarem maior influência da profundidade dos modelos, o que não se verifica na Figura 10 (ISAGHT).

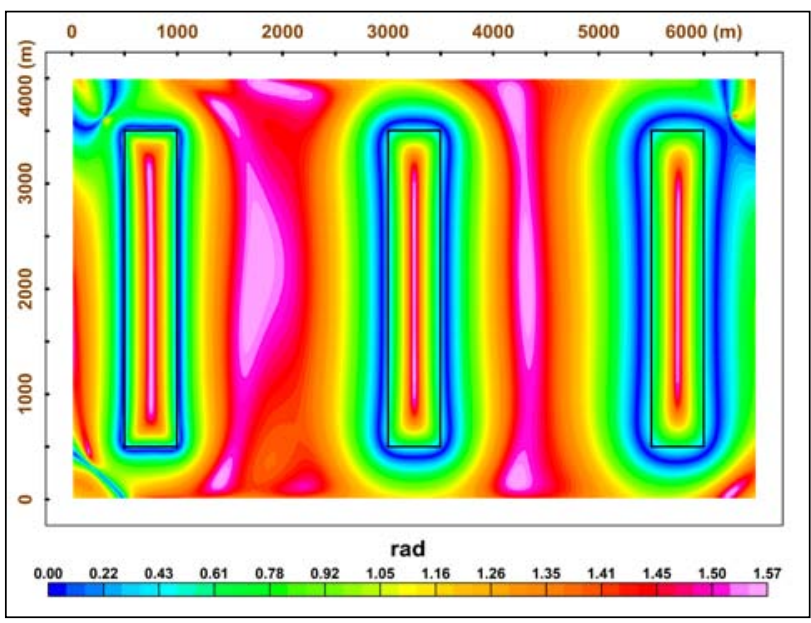

Figura 14 - Theta map calculado com base nos dados da Figura 4.

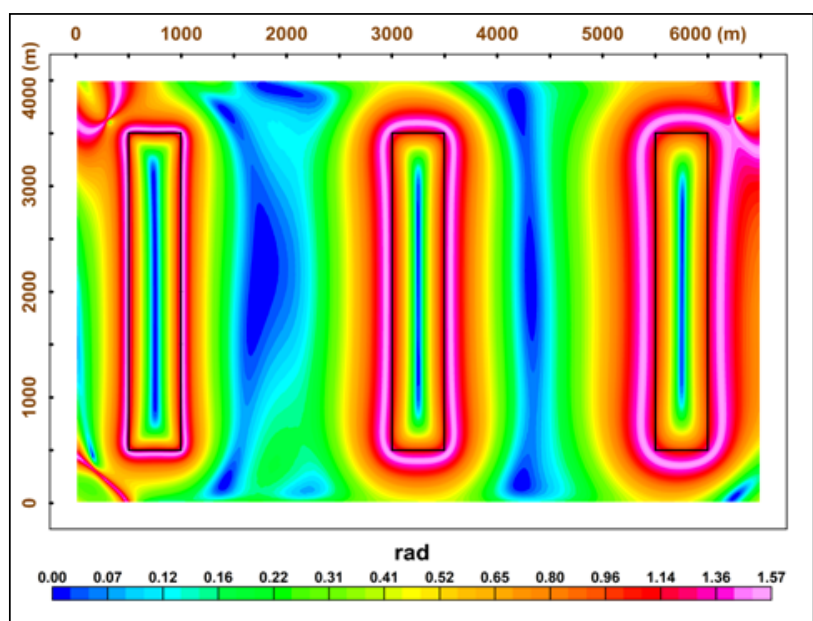

Figura 15 - Mapa da inclinação do gradiente horizontal total (IGHT) calculado com base nos dados da Figura 4.

Theta map e IGHT variam de zero a $+\pi / 2$ (Figs. 14 e 15). Fairhead \& Williams (2006) mencionaram que o intervalo de variação de IGHT é de $-\pi / 2$ a $+\pi / 2$ por não terem considerado os valores absolutos da derivada vertical, em contraposição a definição original de Cooper \& Cowan (2006).

De acordo com a Figura 1, $\theta$ é empregado tanto para calcular ISA quanto Theta map (e.g. Li, 2006b) e os valores absolutos de ISA são iguais a Theta map (e.g. Fairhead \& Williams, 2006), ou seja:

$$
|\mathrm{ISA}|=\text { Theta } \operatorname{map}=\pi / 2-\mathrm{IGHT}
$$

Portanto, concluímos, de acordo com a expressão acima, que basta calcular ISA para obtermos Theta map e IGHT, sem a necessidade de recorrer às respectivas formulações de Wijns et al. (2005) e Cooper \& Cowan (2006). 


\section{Conclusões}

As principais conclusões deste trabalho podem ser assim sumariadas: (i) o método ISA-GHT mostrou resultados satisfatórios, quando aplicado a dados magnéticos reduzidos ao polo, uma vez que realçou igualmente os sinais das bordas dos corpos independentemente de suas profundidades; (ii) Theta map e IGHT são equivalentes e de sinais contrários; (iii) os resultados de Theta map e IGHT são semelhantes aos obtidos por ISAGHT, embora, em nossa proposição, as assinaturas vinculadas às bordas dos prismas se posicionam diretamente sobre seus limites, além de ser pouco influenciada pela profundidade dos modelos, ao contrário do que se verifica nos demais métodos; (iv) Theta map e IGHT podem ser produzidos diretamente de ISA, sem a necessidade de recorrer às respectivas formulações; (v) ISA-GHT é menos sensível a ruídos que GHT-ISA, apesar de ambos utilizarem derivadas de segunda ordem; (vi) Por fim, o método ISA-GHT constitui uma valiosa ferramenta para a interpretação qualitativa de mapas gravimétricos e magnéticos.

\section{Agradecimentos}

Os autores agradecem à Petrobras pelo financiamento da pesquisa, ao Prof. Dr. Carlos Alberto Mendonça (IAGUSP), ao Prof. Dr. Fernando Guspí (Universidad Nacional de Rosario, Argentina) e ao Dr. Oderson A. de Souza Filho (CPRM) pelas discussões e revisão crítica do manuscrito. F.J.F. Ferreira agradece ao CNPq pela Bolsa de Produtividade em Pesquisa.

\section{Referências}

BHASKARA RAO D \& RAMESH BABU N. 1991. A rapid method for three-dimensional modeling of magnetic anomalies. Geophysics, 56(11): 1729-1737.

BHATTACHARYYA BK. 1964. Magnetic anomalies due to prism-shaped bodies with arbitrary polarization. Geophysics, 29(4): 517-531.

CASTRO LG DE, FERREIRA FJF, BONGIOLO ABS, ROMEIRO MAT \& SOUZA J DE. 2010. Realce de anomalias magnéticas do gradiente horizontal total usando a inclinação do sinal analítico. Parte II - Aplicação a dados reais (neste simpósio).

COOPER GRJ \& COWAN DR. 2006. Enhancing potential field data using filters based on the local phase. Computers \& Geosciences, 32(10):1585-1591.

COOPER GRJ \& COWAN DR. 2008. Edge enhancement of potential-field data using normalized statistics. Geophysics, 73(3): H1-H4.

CORDELL L \& GRAUNCH VJS. 1985. Mapping basement magnetization zones from aeromagnetic data in the San Juan Basin, New Mexico. In: Hinze WJ (ed). The Utility of Regional Gravity and Magnetic Anomalies Maps. Society of Exploration Geophysicists, p.181-197. FAIRHEAD JD \& WILLIAMS SE. 2006. Evaluating normalized magnetic derivatives for structural mapping. Expanded Abstracts, Society of Exploration Geophysicists, 25: 845-848.

FAIRHEAD JD, SALEM A, WILLIAMS S \& \$ E. 2008. Magnetic interpretation made easy: The Tilt-Depth-Dip- $\Delta \mathrm{K}$ method. Expanded Abstracts, Society of Exploration Geophysicists, 27: 779-783.

FEDI M \& FLORIO G. 2001. Detection of potential fields sources boundaries by enhanced horizontal derivative method. Geophysical Prospecting, 49(1): 40-58.

FERREIRA FJF, SOUZA J DE, BONGIOLO ABS, CASTRO LG DE \& ROMEIRO MAT. 2010. Enhancing the total horizontal gradient of magnetic anomalies using tilt derivative (in prep.).

LAHTI I \& KARINEN T. 2010. Tilt derivative multiscale edges of magnetic data. The Leading Edge, 29(1): 24-29.

LI X. 2006a. Understanding 3D analytic signal amplitude. Geophysics, 71(2):L13-L16.

LI X. 2006b. On "Theta map: Edge detection in magnetic map" (C. Wijns, C. Perez and P. Kowalczyk, 2005, Geophysics, 70, L39-L43). Discussion and Replay, Geophysics, 71(3): X11-X12.

$\mathrm{LI} X$. 2008. Magnetic reduction-to-the-pole at low latitudes: Observations and considerations. The Leading Edge, 27(8): 990-1002.

MILLER HG \& SINGH V. 1994. Potential field tilt - a new concept for location of potential field sources. Journal of Applied Geophysics, 32(2-3): 213-217.

NABIGHIAN MN. 1972. The analytic signal of twodimensional magnetic bodies with polygonal crosssection: Its properties and use for automated anomaly interpretation. Geophysics, 37(3): 507-517.

NABIGHIAN MN. 1974. Additional comments on the analytic signal of two-dimensional magnetic bodies with polygonal cross-section. Geophysics, 39(1): 85-92.

PHILIPS JD. 2007. Geosoft executables (GX's) developed by the U.S. Geological Survey, Version 2.0, with notes on $\mathrm{GX}$ development from FORTRAN code (Open-File Report 2007-1355), 111p.

PLOUFF D. 1976. Gravity and magnetic fields of polygonal prisms and application to magnetic terrain corrections. Geophysics, 41(4): 727-741.

ROEST WR, VERHOEF J \& PILKINGTON M. 1992. Magnetic interpretation using the $3-D$ analytic signal, Geophysics, 57(1):116-125.

SALEM A, WILLIAMS S, FAIRHEAD JD, RAVAT D \& SMITH R. 2007. Tilt-depth method: A simple depth estimation method using first-order magnetic derivatives. The Leading Edge, 26(12): 1502-1505.

SINGH B \& GUPTASARMA D. 2001. New method for fast computation of gravity and magnetic anomalies from arbitrary polyhedra. Geophysics, 66(2): 521-526.

SOUZA J DE, FERREIRA FJF, BONGIOLO ABS, CASTRO LG DE \& ROMEIRO MAT. PRISMA_GRAV_MAG: um programa para geração de anomalias gravimétricas e magnéticas de corpos prismáticos (em preparação).

VERDUZCO B, FAIRHEAD JD, GREEN CM \& MACKENZIE C. 2004. New insights into magnetic derivatives for structural mapping. The Leading Edge, 23(2): 116-119.

TALWANI M. 1965. Computation with the help of a digital computer of magnetic anomalies caused by bodies of arbitrary shape. Geophysics, 30(5): 797-817.

WIJNS C, PEREZ C \& KOWALCZYK P. 2005. Theta map: Edge detection in magnetic data. Geophysics, 70(4): L39-L43. 